to systemic inflammation, as expressed by increased $\mathrm{IgG}$ and resulting from colonisation with pathogens and irrespective of blood pressure or diabetes status. Conversely, in adult CF patients, CFRD seems to influence arterial stiffness [4]. This discrepancy between adults and children may indicate that the changes in the arterial wall architecture appear only after decades of hyperglycaemic burden.

The present study did not examine whether an intervention, such as intravenous antibiotics, is able to reduce the increase in arterial stiffness; however, a recent study published in abstract form seems to reveal benefits of interventions aiming to reduce inflammation on the cardiovascular system in adult CF patients [10]. It remains unclear whether these interventions completely normalise the increase in arterial stiffness or the vascular changes remain permanently altered. The results of the present exploratory study, together with the results of other studies $[4,10]$, suggest that aggressive and early anti-inflammatory therapies are indicated in CF patients not only to stabilise lung function but also to avoid extrapulmonary complications and to reduce the accelerated vascular ageing process.

A strength of this study is the homogenous and young CF population without other cardiovascular risk factors likely to be encountered in adulthood. Limitations include the lack of other biochemical inflammatory or metabolic markers for cardiovascular risk such as triglycerides and cholesterol.

In conclusion, this study demonstrates haemodynamic alterations in the presence of persisting systemic inflammation already in children suffering from CF. With increasing survival, awareness of these vascular changes is required in order to maintain cardiovascular health in CF patients.

Tobias Buehler*, , Markus Steinmann", Florian Singer", Nicolas Regamey", Carmen Casaulta ${ }^{\#}$, Martin H. Schoeni ${ }^{\#}$, Giacomo D. Simonetti*

*Division of Pediatric Nephrology, and "Division of Pediatric Pulmonology, Children's Hospital, University of Bern, Berne, Switzerland.
Correspondence: G.D. Simonetti, Division of Pediatric Nephrology, Children's Hospital, University of Bern, 3010 Berne, Switzerland. E-mail: giacomo.simonetti@insel.ch

Support Statement: F. Singer received a grant from the European Respiratory Society (Short-Term Research Fellowship 81-2011).

Statement of Interest: None declared.

\section{REFERENCES}

1 Madge S. Growing up and growing older with cystic fibrosis. J $R$ Soc Med 2006; 99: Suppl. 46, 23-26.

2 Cheung YF, Brogan PA, Pilla CB, et al. Arterial distensibility in children and teenagers: normal evolution and the effect of childhood vasculitis. Arch Dis Child 2002; 87: 348-351.

3 Laurent S, Boutouyrie P, Asmar R, et al. Aortic stiffness is an independent predictor of all-cause and cardiovascular mortality in hypertensive patients. Hypertension 2001; 37: 1236-1241.

4 Hull JH, Garrod R, Ho TB, et al. Increased augmentation index in patients with cystic fibrosis. Eur Respir J 2009; 34: 1322-1328.

5 Simonetti GD, Eisenberger U, Bergmann IP, et al. Pulse contour analysis: a valid assessment of central arterial stiffness in children? Pediatr Nephrol 2008; 23: 439-444.

6 Miller MR, Hankinson J, Brusasco V, et al. Standardisation of spirometry. Eur Respir J 2005; 26: 319-338.

7 Singer F, Stern G, Thamrin C, et al. Tidal volume single breath washout of two tracer gases - a practical and promising lung function test. PLoS One 2011; 6: e17588.

8 Moran A, Brunzell C, Cohen RC, et al. Clinical care guidelines for cystic fibrosis-related diabetes: a position statement of the American Diabetes Association and a clinical practice guideline of the Cystic Fibrosis Foundation, endorsed by the Pediatric Endocrine Society. Diabetes Care 2010; 33: 2697-2708.

9 National High Blood Pressure Education Program Working Group on High Blood Pressure in Children and Adolescents. The fourth report on the diagnosis, evaluation, and treatment of high blood pressure in children and adolescents. Pediatrics 2004; 114: 555-576.

10 Hull JH, Garrod R, Ho TB, et al. Do intravenous antibiotics influence arterial stiffness in adults with cystic fibrosis? Thorax 2010; 65: Suppl. 4, A119.

\title{
Monitoring of tobramycin levels in patients with cystic fibrosis by finger-prick sampling
}

\section{To the Editors:}

Chronic pulmonary infection is the major cause of morbidity and premature mortality in patients with cystic fibrosis (CF). The major pathogen for patients with CF is Pseudomonas aeruginosa. Tobramycin, an aminoglycoside antibiotic, is widely used against Gram-negative bacterial infections and is particularly useful for the treatment of $P$. aeruginosa in patients with CF [1]. Intravenous tobramycin has a narrow therapeutic range and monitoring of the drug is required to reduce serious side-effects, such as nephrotoxicity and ototoxicity [2]. Dosage alterations based on the results of drug monitoring can improve efficacy and minimise toxicity. Monitoring currently requires venesection and patients may find finger-prick samples less painful and more acceptable.

Finger-prick blood sampling is routinely performed by patients with diabetes mellitus, is an easy technique to teach and uses 
a)

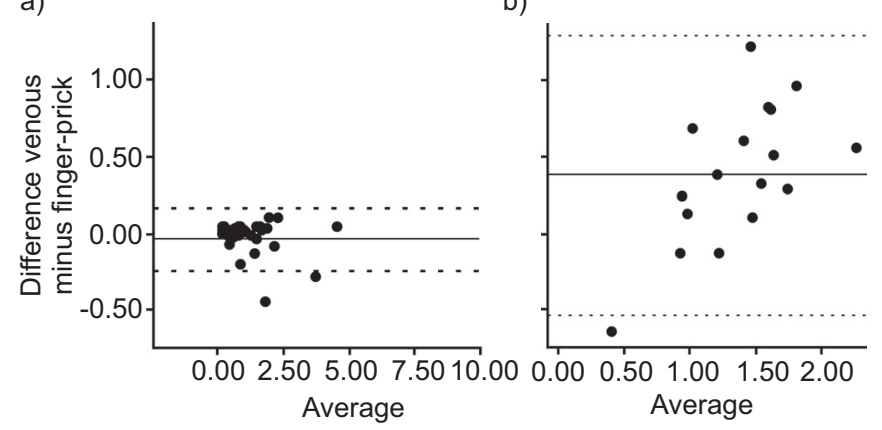

FIGURE 1. Bland-Altman plots of a) pre-dose and b) post-dose differences $\left(\mathrm{mg} \cdot \mathrm{L}^{-1}\right.$ ) between venous and finger-pick tobramycin levels. Dotted lines show $95 \%$ limits of agreement. Solid lines show the mean bias.

inexpensive equipment which is widely available. We have previously shown that finger-prick sampling is a safe and accurate alternative to venous sampling for measurement of tacrolimus levels in children with kidney transplants [3]. Anecdotal experience in children's hospitals suggest that children generally prefer finger-prick sampling to venous sampling, even where topical anaesthetic agents are used for the latter. Studies in adult patients, including solid organ transplant recipients [4] and those receiving anticoagulant therapy [5], have shown a preference for finger-prick over venous blood sampling.

We performed a prospective study to compare the results of tobramycin levels in adult patients with CF measured by finger-prick samples with those from peripheral venous blood using a fluorescence polarisation immunoassay.

Venous blood $(5 \mathrm{~mL})$ and finger-prick blood $(<0.5 \mathrm{~mL})$ samples were collected from patients attending the Manchester Adult Cystic Fibrosis Centre (Manchester, UK) who were receiving i.v. tobramycin therapy. Both samples were taken within a 5-min period. Trough levels were taken immediately prior to the next dose of tobramycin. Peak levels were taken 45 min after the end of the infusion. Patients receiving nebulised tobramycin were excluded from the study.

Tobramycin concentrations were measured on a Cobas Integra analyser using fluorescence polarisation (Roche Diagnostics, Lewes, UK) according to the manufacturer's instructions. The assay requires a $3-\mu \mathrm{L}$ sample, although a dead volume of approximately $100 \mu \mathrm{L}$ is required in the sample cup. The range of the assay is from 0.04 to $10 \mathrm{mg} \cdot \mathrm{L}^{-1}$.

Approval for the study was given by the South Manchester Research Ethics Committee (Manchester, UK) and written informed consent was obtained from all patients.

Statistical analysis was performed using SPSS statistical package version 15.0 (SPSS Inc., Chicago, IL, USA). The agreement between the methods was measured by the conventional Bland-Altman approach [6].

Paired venous blood $(5 \mathrm{~mL})$ and finger-prick blood $(<0.5 \mathrm{~mL})$ samples were collected from 54 patients. Of these 37 were predose levels and 17 were taken 45 min post-dose. The matched pre-dose sample results from one patient were $0.12 \mathrm{mg} \cdot \mathrm{L}^{-1}$ by venous blood sampling and $0.58 \mathrm{mg} \cdot \mathrm{L}^{-1}$ by finger-prick sampling; this would have led to a difference in clinical management with a decrease in the following doses of tobramycin if the finger-prick method had been used. There were no differences in any of the other matched samples that would have led to a change in clinical management. The error rate (clinically significant discrepancy rate) for pre-dose samples was $2.7 \%$ (95\% CI $0.5-13.8 \%$ ) (one out of 37 samples); with no clinically significant differences in post-dose results 0\% (95\% CI 0-18.4\%) (none of the 17 samples). The combined error rate for all samples was 1.9\% (95\% CI 0.3-9.8\%) (one out of 54 samples). Bland-Altman analysis showed good agreement between the two different collection methods with a bias of $-0.03 \mathrm{mg} \cdot \mathrm{L}^{-1}(95 \%$ limits of agreement $-0.24-0.17)$ for predose samples and a bias of $0.38 \mathrm{mg} \cdot \mathrm{L}^{-1}(95 \%$ limits of agreement -0.54-1.30) for post-dose samples (fig. 1).

The first-line i.v. antibiotic regimen for patients with CF and chronic $P$. aeruginosa infection is a combination of $\beta$-lactam and aminoglycoside antibiotics [1]. Tobramycin is the usual aminoglycoside antibiotic of choice in CF because it has greater activity against $P$. aeruginosa, lower rates of resistance among $P$. aeruginosa isolates [7] and is associated with a lesser risk of adverse side-effects than gentamicin [2]. It is important to measure blood levels of tobramycin both to ensure therapeutic dosing and to prevent against toxicity. The current gold standard for monitoring of blood levels of tobramycin is ELISA measurements of tobramycin on peripheral venous blood; however, this requires venesection. We have shown that results of finger-prick sampling are in good agreement with those obtained by i.v. blood sampling. Finger-prick sampling offers a less invasive and potentially more acceptable method than i.v. blood sampling.

Patients with CF often receive several courses of i.v. tobramycin every year and it is important to measure drug levels on each occasion; these patients often have difficult venous access. Care must be taken with the use of central venous catheter samples for measuring tobramycin levels, as these may lead to falsely elevated results [8]. Finger-prick sampling may offer a more acceptable and easier means of obtaining a specimen for analysis than venepuncture. This method could also be used for patients receiving domiciliary i.v. tobramycin. Care, however, should be taken if the patient is using nebulised tobramycin, due to a potential for falsely elevated results caused by contamination on the hands [9].

MADSEN et al. [10] investigated the possibility of measuring tobramycin levels in the saliva of patients with $\mathrm{CF}$ as a noninvasive way of assessing systemic drug levels. However, they found that tobramycin does not distribute into saliva in quantifiable amounts and they concluded that salivary sampling was not a suitable method for the analysis of tobramycin levels in patients with CF.

Prior to i.v. aminoglycoside therapy, the patients should be adequately hydrated and have stable renal function. Care should be taken in patients who may have inherited a predisposition to aminoglycoside toxicity [11], those who have underlying renal or vestibular or ocular problems, or if coadministered with drugs that may have nephrotoxic effects such as colisitin or nonsteroidal anti-inflammatory drugs. In addition to measuring blood tobramycin levels, renal function 
should also be measured in all patients and an annual pure tone audiogram should be considered, especially for patients who receive frequent courses of tobramycin.

While tobramycin levels were slightly lower in the finger-prick samples, the very small mean difference found is only of limited clinical importance. We have previously observed this phenomenon when comparing immunosuppressant levels in finger-prick and venous samples using the same method for measurement [3]. Finger-prick blood (often referred to as capillary blood) is a mixture of arteriolar, venous and capillary blood, although thought to resemble arterial more than venous blood. In addition to blood, samples will also contain small volumes of interstitial and intercellular fluids, possibly accounting for the somewhat lower measured drug levels. Finger-prick blood collection can also be undertaken at home and this may allow the patient to post samples into the laboratory using the regular mail service. This simple technique could save the patient from having to make unnecessary trips to the hospital.

In conclusion, finger-pick sampling can provide a less invasive method than traditional venepuncture for the measurement of blood tobramycin levels in patients with CF.

Andrew Jones*,\#, Judith Beisty*, Diane McKenna*, Debbie Clough* $^{*}$ Kevin Webb*,\#, Julie Morris", ${ }^{\# \text { 厂 }}$ and Brian Keevil ${ }^{+}$ *Manchester Adult Cystic Fibrosis Centre, "Medical statistics, ${ }^{+}$Dept of Biochemistry, University Hospitals South Manchester NHS Foundation Trust, and \#Respiratory Research Group, Faculty of Medical and Human Sciences, The University of Manchester, Manchester Academic Science Health Centre, Manchester, UK.

Correspondence: A. Jones, Manchester Adult Cystic Fibrosis Centre, University Hospitals South Manchester NHS Trust,
Southmoor Road, Manchester, M23 9LT, UK. E-mail: andrew. jones@uhsm.nhs.uk

Statement of Interest: None declared.

\section{REFERENCES}

1 Antibiotic Treatment for Cystic Fibrosis. Report of the UK Cystic Fibrosis Trust Antibiotic Working Group. 3rd Edn. Cystic Fibrosis Trust, 2009.

2 Smyth AR. Minimizing the toxicity of aminoglycosides in cystic fibrosis. J R Soc Med 2010; 103: Suppl. 1, s3-s5.

3 Webb NJ, Roberts D, Preziosi R, et al. Fingerprick blood samples can be used to accurately measure tacrolimus levels by tandem mass spectrometry. Pediatr Transplant 2005; 9: 729-733.

4 Merton G, Jones K, Lee M, et al. Accuracy of cyclosporine measurements made in capillary blood samples obtained by skin puncture. Ther Drug Monit 2000; 22: 594-598.

5 Woods K, Douketis JD, Schnurr T, et al. Patient preferences for capillary $v s$ venous INR determination in an anticoagulation clinic: a randomized controlled trial. Thromb Res 2004; 114: 161-165.

6 Bland JM, Altam DG. Statistical methods for assessing agreement between two methods of clinical measurement. Lancet 1986: 1; 307-310.

7 Pitt T, Sparrow M, Warner M, et al. Survey of resistance of Pseudomonas aeruginosa from UK patients with cystic fibrosis to six commonly prescribed antimicrobial agents. Thorax 2003; 58: 794-796.

8 Mogayzel PJ, Pierce E, Mills J, et al. Accuracy of tobramycin levels obtained from central venous access devices in patients with cystic fibrosis is technique dependent. Pediatr Nurs 2008; 34, 6: 464-469.

9 Struthers SL, Nicolson T, Jones G, et al. Falsely elevated serum tobramycin levels in a patient receiving nebulised tobramycin. J Cyst Fibros 2002; 1: 146-147.

10 Madsen V, Lind A, Rasmussen $\mathrm{M}$, et al. Determination of tobramycin in saliva is not suitable for therapeutic drug monitoring of patients with cystic fibrosis. J Cyst Fibros 2004; 3: 249-251.

11 Mulrennan SA, Helm J, Thomas RB, et al. Aminoglycoside ototoxicity susceptibility in cystic fibrosis. Thorax 2009; 64: 271-272.

DOI: $10.1183 / 09031936.00176611$

\section{Noninvasive ventilation in cystic fibrosis: the Italian physiotherapists' point of view}

\section{To the Editors:}

A physiological rationale has been demonstrated for the use of noninvasive ventilation (NIV) in patients with cystic fibrosis (CF) [1]; however, there is little evidence [2] to support NIV as a routine clinical treatment in people with $\mathrm{CF}$, especially for those with advanced lung disease. Possible explanations include the lack of clearly validated criteria to propose NIV, controversies with regard to the optimal ventilatory modes and settings, scepticism with regard to long-term efficacy [3], poor acceptance by some patients and, above all, lack of familiarity with this technique among health workers. Instead, respiratory physiotherapists are often involved in the NIV management with different skills and tasks [4]. The aim of this study was to survey and evaluate the role and competencies of Italian physiotherapists involved in NIV management in CF centres and to understand the rationale of NIV as perceived by them. A semi-structured online questionnaire consisting of 31 closed and nine open-ended questions was sent to the Italian Group of Physiotherapists $(n=63)$ belonging to the Italian Society for the Study of Cystic Fibrosis, between March and April 2010. Respondents ( $n=42,67 \%)$ worked in 24 CF centres (18 centres and six clinics), which had a total of $4,064 \mathrm{CF}$ patients at the time of the study, $96(2.4 \%)$ of those were using NIV. NIV was the therapeutic choice used in $63 \%$ of those centres and 28 (93\%) out of 30 physiotherapists were involved 